REVISION DE LA LITERATURA 


\title{
CULTURA Y EDUCACION: HACIA UNA PEDAGOGIA DIFERENCIAL
}

\author{
Chrístian Hederich Martínez \\ Universidad Pedagógica Nacional (CIUP) \\ Asesor MEN-Saber \\ Angela Camargo Uribe \\ Universidad Pedagógica Nacional (CIUP)
}

\section{Introducción}

En 1991, el Ministerio de Educación Nacional, a través del Sistema Nacional de Evaluación de la Calidad de la Educación, examinó algunas dimensiones de la calidad de la educación en trece departamentos del territorio nacional. Los resultados obtenidos indican que existen importantes diferencias en el logro de los alumnos asociadas con los departamentos que habitan. Más específicamente, se encontró, que mientras los estudiantes de Bogotá y la zona cafetera mostraban altos logros relativos en todas las pruebas y grados, lo contrario sucedía para los estudiantes de la costa Atlántica ${ }^{1}$.

Adicionalmente, el estudio indagó acerca de las variables que podrían ayudar a explicar las diferencias en el logro académico. Los resultados son importantes, aun cuando, como es usual en este tipo de estudios, no alcanzan a explicar completamente las diferencias en el logro.

El presente artículo centra su objeto en el estudio de una variable que podría llegar a explicar buena parte de estas diferencias: la desarticulación entre el contexto cultural y el modelo educativo. Desarrollaremos primero esta idea. Hecho esto, exploraremos un modelo teórico para la comprensión de las influencias de la cultura en la cognición del sujeto. Pasaremos entonces a la exposición de algunas implicaciones educativas 1) en el nivel de la implementación de modelos educativos, 2) en el nivel de la formulación de políticas, y 3) en el nivel de la interacción educativa básica: la del alumno en su microambiente del aula.

\section{La contradicción cultura-modelo educativo}

Es un lugar común afirmar que los modelos educativos en América Latina no corresponden a las características culturales de la población. Magendzo (1985) nos refiere sus observaciones entre los indígenas guatemaltecos de la siguiente manera:

El niño y la niña guatemaltecos desarrollan un sinnúmero de habilidades, destrezas, valores y actitudes: ahí están los coloridos, hermosos y multivariados bordados; los niños vendiendo en los mercados, sumando y restando intuitivamente. En la escuela el fracaso escolar es alarmante: de 208.622 alumnos que ingresan a primer grado de 1973, sólo llegaron a concluir el nivel 49.243.

Los puntos en donde se evidencia esta contradicción son múltiples y van, desde los modelos didácticos utilizados por los maestros hasta las estructuras de poder inherentes a la institución educativa, tal y como la concebimos actualmente. Esta contradicción se hace

\footnotetext{
${ }^{1}$ MINISTERIO DE EDUCACION NACIONAL. Saber-Sistema Nacional de Evaluación de la Calidad de la Educación. Primeros resultados: Matemáticas y Lenguaje en la Básica Primaria. Santafé de Bogotá, 1992. 
evidente si observamos que los modelos educativos utilizados fueron diseñados originalmente para grupos sociales cultivados y de altos ingresos (Lennon, 1988).

Como es obvio, los casos en donde no se observa esta contradicción muestran resultados opuestos: en estudios con niños de escuelas rurales, Dodendoff encontró que la coincidencia entre los valores enfatizados en la escuela y aquellos inculcados por la comunidad se reflejaba de manera significativa en los altos niveles de logro escolar en estos niños, a través de la construcción de "un mundo seguro y estable para que los niños crecieran". (Dodendoff, 1983; citado por Magendzo 1985).

Los casos en que los parámetros culturales apoyan positivamente el logro educativo son francamente impactantes: entre los inmigrantes indochinos en los Estados Unidos, se observó que, a pesar de las evidentes desventajas lingüísticas y económicas, los jóvenes alcanzaban rápidamente puntajes sobresalientes a nivel nacional, particularmente en las áreas de matemáticas y ciencias. Parte de la explicación para este hecho parece residir en la concepción de educación que la familia indochina mantiene. Para estos hogares, la educación de los pequeños es responsabilidad fundamental de la familia en su conjunto, $y$ no de la institución escolar. Se crea así una red de apoyo al logro académico del pequeño, en la que toman parte los padres y los hermanos mayores trabajando juntos (Kaplan, Choy y Whitmore, 1992).

\section{De la cultura a la educación}

Dentro de las muchas posibilidades de conceptualizar el objeto de la cultura, es interesante anotar la aportada por el llamado "Marco de Referencia Eco-cultural". (Laboratory of Comparative Human Cognition, 1986). De acuerdo con esta concepción, un grupo humano cualquiera, localizado en una zona geográfica determinada enfrenta inicialmente un contexto de tipo físico y ecológico determinado que limita sus posibilidades de actividad económica. Basta observar, por ejemplo, que un grupo humano localizado en una zona desértica desarrolla actividades de supervivencia no relacionadas con la agricultura.

En el desarrollo de sus actividades de sobrevivencia, el grupo establece una forma de organización social apropiada y adaptada para sus propósitos. Se establecen entonces formas de organización social y estructuras de parentesco adecuadas, que son perpetuadas por prácticas de crianza construidas al interior de la cultura. Estos cuatro factores: medio ambiente físico, actividad económica, organización social y prácticas de crianza, se unen en la constitución de un referente simbólico que constituye el universo expresivo del sujeto.

En resumen, la interacción de condiciones ambientales ha creado una visión particular del mundo y de sí mismo: un sentido del "yo" y del "mundo que me rodea". Un ejemplo de esta diferencia estructural, relacionado con la cultura, es el documentado por Guttman en 1967 para los campesinos de origen maya en México. Frente a la pregunta de si les agradaba su trabajo, los individuos contestaban de forma evasiva e imprecisa. Finalmente se observó que, al no diferenciar entre "yo" y "mi trabajo' , una pregunta fundada en esta distinción no tenía el menor sentido (Guttman, 1967; citado por Price-Williams, 1980).

Ahora bien, a través del proceso ya descrito, cada medio eco-cultural específico determina un cierto conjunto de comportamientos que resultan especialmente adaptados al contexto. En sociedades de cazadores nómadas, por ejemplo, la importancia de las habilidades perceptivas individuales es crucial, mientras que otro tipo de habilidades 
puede no ser tan importante (Witkin y Berry, 1975). El proceso educativo de inicio cuando el grupo cultural intenta desarrollar, en los jóvenes, este tipo particular de habilidades, incluso en detrimento de otras que podrían ser consideradas "indeseables" para el contexto.

De forma más específica, la investigación ha documentado la influencia del contexto cultural en:

1) el desarrollo de sensotipos particulares, como el caso de las culturas africanas occidentales que se apoyan más en informaciones propioceptivas y auditivas, que en informaciones visuales, tan importantes para la cultura occidental (Wober, 1967 citado por Price-Williams, 1980),

2) la construcción de conceptos, con diferentes grados de abstracción o concreción. Un ejemplo es el concepto de "distancia a un determinado punto" entre los tahitianos, para quienes es importante considerar, en la definición, informaciones tales como el medio usado para llegar al punto en cuestión y el motivo por el cual la noción de "distancia" surgió en la conversación. (Levy, 1973 citado por Price-Williams, 1980),

3) la generación, a través de prácticas específicas de socialización, de determinados comportamientos sociales, desconociendo o estigmatizando otros como el caso de los israelitas de origen asiático entre quienes se observa una mayor tendencia hacia la sumisión a la autoridad, en comparación con compatriotas suyos de origen occidental (Preale y cols., 1970; citado por Price-Williams 1980).

\section{Estilos cognitivos}

Hasta este punto, hemos mostrado cómo, dentro de un grupo humano, se generan universos simbólicos específicos que constituyen su cultura, y cómo son transmitidos y perpetuados a las nuevas generaciones privilegiando la constitución de ciertas habilidades básicas. El proceso, a nivel individual, puede ser entendido, desde la ciencia cognitiva, como la constitución de un estilo particular de procesar la información proveniente del entorno. Esto es, un estilo cognitivo.

Por definición, cada estilo cognitivo cumple con la característica de ser una modalidad de procesamiento especialmente adecuada para las exigencias del entorno eco-cultural específico. Esto significa que, al tiempo que favorece cierto grupo de habilidades, desfavorece otras. En este sentido, cada estilo presenta una serie de ventajas y desventajas propias. Esto implica que no existe un estilo cognitivo pretendidamente superior: para ciertas tareas, ciertos estilos pueden ser más apropiados que otros, para otras, la situación puede ser contraria.

Existen diferentes operacionalizaciones del concepto "estilo cognitivo". Entre ellas, pueden mencionarse las polaridades de "impulsividad/reflexividad", y "dependencia/independencia del medio"2. En general, las diferentes concepciones coinciden en afirmar que existe, en cada individuo, una estabilidad respecto de su funcionamiento cognitivo. Cierto tipo de información es permanentemente privilegiada en detrimento de otra, y cierto tipo de procesamiento de la misma es ejecutado de forma más frecuente. Esta característica permite desarrollar una alta efectividad para la resolución de algunos problemas, en general relacionados con el contexto ambiental, y disminuye la

\footnotetext{
${ }^{2}$ Más conocida como dependencia-independencia de campo. Esta última denominación es una traducción literal del concepto field dependence.
} 
competencia en la resolución de otros, más alejados del contexto. Igualmente, favorece ciertos tipos de contenido de aprendizaje, dificultando otros.

Dentro de este contexto teórico, la contradicción cultura/modelo educativo cobra una nueva dimensión. En efecto, los estilos desarrollados por el individuo como parte de su herencia cultural son, de hecho, relevantes para su adaptación. Este estilo es fortalecido de forma cotidiana y permanente dentro del contexto y, en ese sentido, cualquier intento educativo contradictorio a la cultura es desechado de múltiples formas.

En general, dentro de las muchas formas de rechazo a un modelo educativo contradictorio al contexto cultural, se destaca un rechazo que podemos llamar "de tipo activo", en el que existe una abierta y explícita desadaptación del sujeto al modelo, que se manifiesta usualmente en problemas de disciplina y rendimiento, y que se traduce finalmente en altos índices de deserción del sistema escolar.

Sin embargo, un tipo de rechazo más frecuente, y más adaptado si se quiere, es de tipo "pasivo". En este, el estudiante aprende, sólo superficialmente, aquellas conductas que le hacen salir airoso de la situación concreta de evaluación educativa, sin que estos aprendizajes resulten significativos para su vida cotidiana. Esto constituye uno de los resultados más preocupantes aportados por el Sistema de Evaluación: en la resolución de las pruebas de matemáticas, por ejemplo, los estudiantes mostraron una muy alta habilidad para la resolución de algoritmos y una muy baja capacidad para la resolución de los problemas que involucraban estos algoritmos. En resumen, el estudiante ha aprendido académica y descontextualizadamente, información que no comprende ni puede utilizar (MEN-SABER, 1992).

Parte de esta situación puede estar sucediendo en las costas colombianas. En efecto, si suponemos un conjunto de rasgos comunes a las culturas localizadas en zonas costeras, esta contradicción entre el modelo educativo y el contexto cultural ya se ha evidenciado. Entre las poblaciones de estudiantes hawaianos, por ejemplo, la importancia que la cultura asigna a la colaboración intra-familiar es tan grande, que los estudiantes parecen prestar más atención a sus compañeros de clase que a sus profesores. Esta conducta es interpretada por los profesores como de "falta de interés por las labores académicas" (Gallimore, Boggs y Jordan, 1974; citados por Magendzo, 1985).

Este rasgo cultural que asigna gran importancia a la interacción personal podría ser común en varios grupos localizados en las zonas costeras. Otros elementos comunes en estas culturas podrían aventurarse: Smith (1948) observó en estudiantes de las islas Hébridas, que su concepción del tiempo excluía la noción de "de prisa". Para ellos, era muy difícil comprender la necesidad de realizar una tarea en el menor tiempo posible: tiempo era lo que tenían, no era necesario "ahorrarlo".

\section{Implicaciones para la educación}

Las aplicaciones del enfoque expuesto para el sector educativo son múltiples y se dirigen a diferentes niveles. En el contexto del presente artículo se enumerarán algunas implicaciones a nivel de 1) modelo educativo, 2) decisiones de política educativa, y 3) relación docente-alumno.

El nivel de modelo educativo puede ser el de implicaciones más profundas y estructurales. La escuela, tal y como la concebimos en la actualidad, reproduce espacios 
de trabajo individualistas y competitivos: los exámenes deben resolverse de forma solitaria y en el menor tiempo posible, el trabajo en grupo es casi inexistente, la estructura de autoridad es vertical e inapelable, y el trato es formal y distante entre profesores y alumnos. Esta escuela reproduce, en suma, un ambiente social occidental, urbano e industrializado, fuertemente contrastado con el que podemos encontrar en zonas costeras, por ejemplo. Para estas zonas, es evidente la necesidad de búsqueda de modelos educativos más apropiados que, seguramente, manejen de forma diferente el tiempo, y hagan de la relación interpersonal la estrategia más poderosa de aprendizaje. Es fundamental realizar investigaciones que arrojen más información para estos eventuales modelos.

En el nivel de política educativa, se destacan las recomendaciones elaboradas por diferentes estudios acerca de la adecuación de los programas curriculares a los niveles regionales y locales (MEN-SABER, 1992; McGinn y cols., 1992). En este sentido, es fundamental preservar un equilibrio adecuado entre los contenidos propios de las regiones, y los contenidos mínimos comunes al nivel nacional.

Tal y como se ha justificado, la absoluta priorización de aprendizajes externos, ajenos al contexto, es altamente inconveniente. Cada sujeto requiere de ciertos aprendizajes vitales para su contexto cotidiano ya que, finalmente, y con alta probabilidad, este contexto representará su entorno simbólico, económico y laboral del futuro. En el nivel de política, parte de la solución puede ser adelantada con procesos de regionalización de los programas curriculares. Sin embargo, es incuestionable la importancia de lograr un cierto grupo de aprendizajes básicos y comunes a todas las regiones, entre los cuales se destacan los relacionados con lectoescritura y matemáticas. Este grupo de aprendizajes pueden ser adecuados regionalmente con estrategias didácticas y materiales específicos, que trataremos en el nivel pedagógico.

Las implicaciones educativas en el nivel pedagógico son las que pueden resultar más difícil de conceptualizar, y a la vez las de mayor impacto en el logro. En efecto, la experiencia ha demostrado que no podremos lograr ningún avance en la calidad de la educación, si la adecuación del modelo educativo al contexto cultural no se hace a partir de la práctica pedagógica del maestro. Pero, ¿cómo puede lograrse tal cosa?

La solución puede vislumbrarse desde la teoría. En efecto, se sabe que cada estilo cognitivo desarrolla, desde sus potencialidades, ciertos procedimientos para el logro de aprendizajes difíciles. Estos procedimientos característicos constituyen la mejor vía para que el individuo de un estilo dado lleve a cabo su aprendizaje exitosamente. En este sentido es fundamental para el maestro reconocer las características cognitivas de sus alumnos. A partir de esta información, podrá diseñar múltiples estrategias de presentación de la información, acordes con el contexto.

Un ejemplo de aplicación de la información cognitiva es el señalado por los datos que muestran diferencias cognitivas entre las poblaciones rurales y urbanas en el aprendizaje matemático. De acuerdo a lo encontrado, los estudiantes del sector rural presentan mejores resultados en el desarrollo de la estructuración del espacio, que sus congéneres de sectores urbanos, mostrando al mismo tiempo menos habilidad que éstos en lo relacionado con operaciones de tipo numérico discontinuo (Hederich y cols., 1988) ${ }^{3}$. Esta información puede ser utilizada para la definición de modelos didácticos fundamentados

\footnotetext{
${ }^{3}$ Existen fuertes indicios que apoyarán estos datos, en el contexto del Sistema Nacional de Evaluación de la Calidad de la Educación.
} 
en manejos continuos para los sectores rurales y discontinuos para sectores urbanos, que representa una tarea relativamente sencilla.

Dentro de estos modelos pedagógicos apropiados a la región hay un punto que no debe pasarse por alto, y es el de las diferencias, ya no inter-culturales, sino simplemente individuales. Efectivamente, no podemos olvidar que el estilo cognitivo es una forma de funcionamiento de naturaleza individual y que, dentro de cada grupo de estudiantes, existen diferencias importantes. Así, el papel del maestro tiene que dirigirse hacia la potenciación, en cada alumno, de sus mejores habilidades, para la superación de sus deficiencias. Estamos hablando, en suma, de una pedagogía diferencial.

Es difícil aportar grandes precisiones acerca de lo que podría llegar a ser una pedagogía que respete las diferencias individuales. Una posibilidad está en propiciar modalidades de interacción entre los estudiantes de forma que, entre ellos, se solucionen las dificultades de cada uno: a veces el mejor maestro es un estudiante. Otra posibilidad está dada por un manejo diferencial del tiempo de instrucción y del tiempo y la modalidad de evaluación. La factibilidad de este tipo de soluciones sólo puede ser esclarecida a través de la experiencia en el aula de un maestro consciente de las diferencias entre sus alumnos. A partir de maestros así, puede llegar a construirse una sociedad más consciente y respetuosa de las diferencias étnicas, ideológicas e individuales. Ese es, en suma, el ideal social que estamos buscando.

\section{REFERENCIAS}

DODENDOFF, D.M. (1983). A Unique Rural School EnvironmenL En: "Ps chology in the Schools". Creighton University.

GALLIMORE, R., BOGGS, J.W. y JORDAN, C.E. (1974). Culture, Belzavior and Education: A Study of Hawaiian-Americans. Beverly Hills. California. Sage.

GUTTMAN. D. (1967). Qn Cross-cultural Studies as a Naturalistic Approach in Psychology. En: "Human Development", 10, 187-198.

HEDERICH. C. y cols. (1988). Estructuración Espacial en Medio Rural. Bogotá: Universidad Externado de Colombia.

LABORATORY OF COMPARATIVE HUMAN COGNITION, (1986). Culturra e Inteligencia. En: R. STERNBERG (Ed.) “Inteligencia Humana”. Vol. III. Barcelona: Paidós, 1988.

LENNON, O. (1988). Variaciones culturales, Estilos Cognitivos y Educación en América Latina. En: "Revista Perspectivas", Unesco, Vol. XVII. No. 3.

LEVY, R. (1973). Tahitians: Mind and Experience in the Society Islands. Chicago: Universitv of Chicago Press.

MAGENDZO. J.A. (1985). Calidad de la Educación y sus Relaciones con la Cultura: Síntesis de una Investigación en un área indígena de Guatemala. En: "La Educación. Revista Interamericana de Desarrollo Educativo", 96, 32-48.

McGINN, N. y cols. (1992). La repitencia de grado en la Escuela Pública Colombiana. Santafé de Bogotá; Harvard Institute for International Development -Education Group-. 
PRICE-WILLIAMS. D.R. (1980). Por los Senderos de la Psicología Intercultural. México: Fondo de Cultura Económica.

PREALE, I. y cols. (1970). Perceptual Articulation and Task Effectiveness in several Israel Subcultures. En: "Journal of Personality and Social Psychology". 15, 190-195.

SMITH. C.M.. (1948). Mental testing of Hebridean children in Gaelic and English. University of London Press.

WITKIN H.A. y BERRY, J.M. (1975). Psychological differentiation in cross-cultural perspective. En: "Journal of aoss-cultural Psychology". 6. 4-87.

WOBER. M. (1967). Adapting Witkin's field independence theory to accomodate new information from Africa. En: "British Journal of Psychology", 58, 29-3 8. 